Nonlinear Processes in Geophysics (2005) 12: 311-320

SRef-ID: $1607-7946 / \mathrm{npg} / 2005-12-311$

European Geosciences Union

(c) 2005 Author(s). This work is licensed

under a Creative Commons License.

\title{
Forced versus coupled dynamics in Earth system modelling and prediction
}

\author{
B. Knopf ${ }^{1}$, H. Held ${ }^{1}$, and H. J. Schellnhuber ${ }^{1,2}$ \\ ${ }^{1}$ Potsdam Institute for Climate Impact Research (PIK), P.O. Box 601203,14412 Potsdam, Germany \\ ${ }^{2}$ Tyndall Centre for Climate Change Research, Norwich, UK
}

Received: 20 October 2004 - Revised: 10 February 2005 - Accepted: 11 February 2005 - Published: 17 February 2005

\begin{abstract}
We compare coupled nonlinear climate models and their simplified forced counterparts with respect to predictability and phase space topology. Various types of uncertainty plague climate change simulation, which is, in turn, a crucial element of Earth System modelling. Since the currently preferred strategy for simulating the climate system, or the Earth System at large, is the coupling of sub-system modules (representing, e.g. atmosphere, oceans, global vegetation), this paper explicitly addresses the errors and indeterminacies generated by the coupling procedure. The focus is on a comparison of forced dynamics as opposed to fully, i.e. intrinsically, coupled dynamics. The former represents a particular type of simulation, where the time behaviour of one complex systems component is prescribed by data or some other external information source. Such a simplifying technique is often employed in Earth System models in order to save computing resources, in particular when massive model inter-comparisons need to be carried out. Our contribution to the debate is based on the investigation of two representative model examples, namely (i) a low-dimensional coupled atmosphere-ocean simulator, and (ii) a replica-like simulator embracing corresponding components.

Whereas in general the forced version (ii) is able to mimic its fully coupled counterpart (i), we show in this paper that for a considerable fraction of parameter- and state-space, the two approaches qualitatively differ. Here we take up a phenomenon concerning the predictability of coupled versus forced models that was reported earlier in this journal: the observation that the time series of the forced version display artificial predictive skill. We present an explanation in terms of nonlinear dynamical theory. In particular we observe an intermittent version of artificial predictive skill, which we call on-off synchronization, and trace it back to the appearance of unstable periodic orbits. We also find it to be governed by a scaling law that allows us to estimate the probability of artificial predictive skill. In addition to artificial pre-
\end{abstract}

Correspondence to: $\mathrm{B}$. Knopf

(knopf@pik-potsdam.de) dictability we observe artificial bistability for the forced version, which has not been reported so far. The results suggest that bistability and intermittent predictability, when found in a forced model set-up, should always be cross-validated with alternative coupling designs before being taken for granted.

\section{Introduction}

In the climate modelling community it is common practice to establish a modular structure, consisting of ecosphere, biosphere, vegetation, ocean, atmosphere, etc., that builds up an Earth System Model (cf. the Climate System Model project CSM Boville and Gent, 1998). Some of these components are also modelled by external forcing, described from observed data. This is done e.g. in the Atmospheric Model Intercomparison Project AMIP (Gates et al., 1992), where an atmospheric general circulation model (AGCM) is constrained by realistic sea surface temperature and sea ice and the output is used for diagnostic research. Although this experiment is not meant to be used for climate change predictions, diagnostic subprojects have been established, though it is not quite clear to what extent the forced AGCM output is comparable to the system with complex ocean-atmosphere feedbacks. These coupled systems are investigated e.g. in the Coupled Model Intercomparison Project CMIP (Meehl et al., 2000; Covey et al., 2003a). The comparison of coupled ocean-atmosphere models with simulations using prescribed sea surface temperatures shows that there are indeed some important differences concerning e.g. temperatures near the pole and tropical precipitation (Covey et al., 2003b). Other publications mention a strong effect of the coupling on the midlatitude variability of the ocean-atmosphere system (Barsugli and Battisti, 1998) or on the decadal variability of oceanic variables in the North Pacific (Pierce et al., 2001).

Hence, the subject of investigation is the effect of prescribing a module through data instead of implementing the dynamical module. This has already been investigated by Wittenberg and Anderson (1998), but here we will focus on po- 


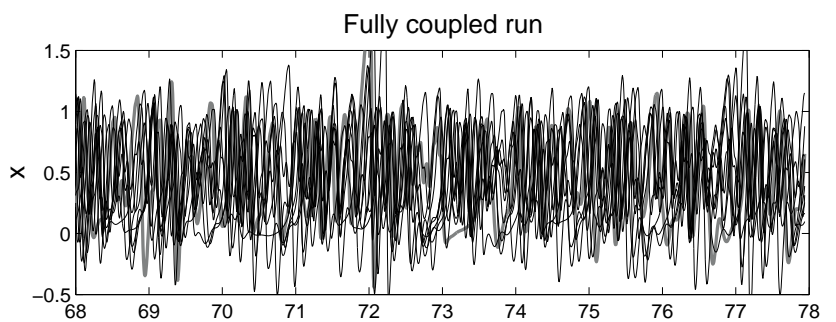

Forced run

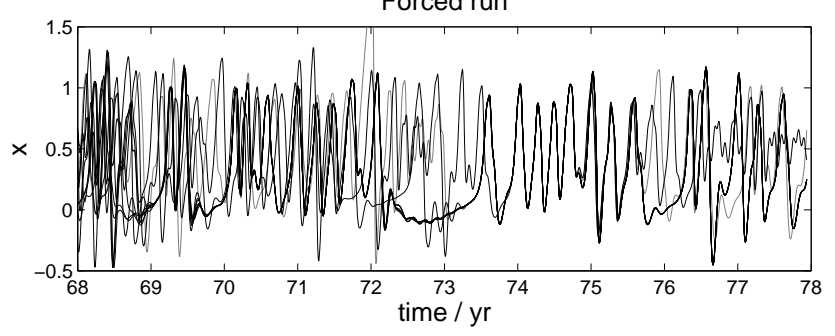

Fig. 1. Comparison of the fully coupled system (top) and the forced system (bottom). A fully coupled run is taken as reference trajectory. Additional to the reference trajectory, in each subfigure there are runs from slightly varying initial conditions in atmospheric coordinates. In the upper figure the curves are from a fully coupled run. In the lower figure, the trajectories are forced by the ocean from the reference trajectory. In this figure, we have reproduced a major finding by Wittenberg and Anderson (1998).

tential constraints preventing the consistency of forcing and coupling. With this paper we want to emphasize that when forcing one module by another instead of coupling the two components, one has to keep in mind that inherently nonlinear phenomena can occur that lead to qualitatively different features than expected. This type of analysis that we are undertaking can be assigned to many other cases of investigating forced versus coupled model runs.

For our study we use a conceptual model which, compared to more sophisticated climate models like GCMs, has the advantage that the model itself as well as the output can transparently be analysed along the lines of dynamical systems' theory. This makes it easier to realise path continuation of solutions in parameter space. The results presented in this paper reveal the underlying mechanisms for certain artificial phenomena produced in forced systems.

From the knowledge of the mechanism we then conclude that those shortfalls of forced models are generic. Therefore we do not so much suggest to perform path continuation for GCMs as well (although it has successfully been implemented also for GCMs, Dijkstra, 2000) but we understand the following sections as a motivation to cross-validate a certain class of forced GCM results by alternative coupling designs in quite a classical manner.

The structure of the paper is as follows: The coupled ocean-atmosphere model we are analysing is described in Sect. 2 and the phenomenon of locking for coupled and forced trajectories is presented. In Sect. 3 the mathematical background for replica systems is introduced. In Sect. 4 we analyse the model in dependence on its parameters and highlight some fundamental differences between forcing and coupling. The role of unstable periodic orbits concerning the locking phenomenon is also investigated. In Sect. 5 we determine the statistics of the locking and deduce a power law scaling for the length of the locking phases. The paper will finish with the conclusions in Sect. 6.

\section{Coupled and forced model}

To investigate the difference between a forced and a fully coupled set-up, a coupled atmosphere-ocean system is chosen because the predictability of the Earth's climate depends strongly on the variability induced by the interaction of these two components. As a very instructive example of the coupled atmosphere-ocean system, the following low-order model is examined:

$$
\begin{aligned}
\dot{x} & =-y^{2}-z^{2}-a x+a(F+\sin (2 \pi \gamma t)) \\
\dot{y} & =x y-c y-b x z+G+\alpha p \\
\dot{z} & =x z-c z+b x y+\alpha q \\
\dot{p} & =-\omega q-\beta y \\
\dot{q} & =\omega p-\beta z
\end{aligned}
$$

with $a=0.125, F=3.5, c=0.5, b=4, \alpha=\beta=0.1, G=0.25$, $\gamma=10 / 365.25, \omega=2 \pi \gamma / 4$, where $\gamma$ is a scaling factor with one unit of system's time referring to 10 days.

This model is taken from Wittenberg and Anderson (1998). The atmosphere system model (Eqs. 1-3) is a potentially chaotic Lorenz system (Lorenz, 1984), that describes the midlatitude quasi-geostrophic flow. While $t$ represents the time, the variable $x$ represents the intensity of the westerly wind current or the meridional temperature gradient. The variables $y$ and $z$ are the amplitudes of the sine and cosine components of a large travelling wave, which transports heat poleward. $F$ and $G$ are forcing terms based on the average north-south temperature contrast and the earthsea temperature contrast, where the seasonal variation of $F$ is expressed through the sine. The ocean system is a simple harmonic oscillator, with an oscillation frequency $\omega$ of four years, where $p$ and $q$ represent zonal asymmetries in sea surface temperature. The coupling between ocean and atmosphere proceeds through the interaction of these asymmetries with the model atmosphere's eddy field ( $y$ and $z$ ).

Wittenberg and Anderson (1998) carried out two different sets of simulations. One set of simulations represents the outcome of the fully coupled system with little variation in the initial state vectors. In the other set the output of the ocean from one special run is used to force the atmosphere. Again this is undertaken for slightly perturbed initial conditions. So there are two ensembles: one from a fully coupled system and one from a forced system that includes no feedback from the atmosphere to the ocean.

As can be seen from Fig. 1, which was reproduced from Wittenberg and Anderson (1998), the forced ensemble is more compact, but does not mirror the true solution. Furthermore, Wittenberg and Anderson (1998) show that the statis- 
tics of the forced variability, like spatial and temporal distributions, are significantly different from those of coupled variability. For modelling issues this means that a prescribed forcing (e.g. prescribing the sea surface temperature) cannot emulate the fully coupled system. The interesting effect of the forcing is that all trajectories sometimes lock on the true solution for a short time and then separate again, so partial synchronization, so called "locking" can be observed. One can conclude from this that on the one hand fully coupled and forced systems do not show the same behaviour but on the other hand that in truly forced systems there may exist a region in phase space, where predictability is very high. The question to be followed is what mechanism is responsible for the locking phenomenon and what types of coupling show such behaviour.

\section{Mathematical framework}

In order to explain the locking phenomenon, a stability analysis of the system appears the most natural approach. In the tradition of Wittenberg and Anderson (1998) and Smith et al. (1999), one would expect that the local linear stability properties govern the observed phenomenon. Empirically, however, we find that the explanation for locking given in Wittenberg and Anderson (1998) does not hold. We find that locking shows no correlation to the trajectory's residence in the "locking region" identified in Wittenberg and Anderson (1998) and, in particular, that locking persists an order of magnitude longer than the trajectory resides in the locking region. Contrary to a local linear stability analysis, we will relate the locking period to extended invariant manifolds, emerging from the nonlinear dynamics of the system. This will allow us to introduce meaningful time-averaged characteristics. To frame a discussion of potential nonlinear causes of locking, we follow the concepts of Pecora and Carroll (1990) and Pecora et al. (1997). Different from Fig. 1, where we looked at a set of several forced trajectories, we investigate here just the fully coupled run and one forced run. The forced system can be written as a so-called replica system (Pikovsky et al., 2001), where a replica of one or more equations is made. Together with Eqs. (1)-(5) we have a replica system of the following form:

$\dot{x}^{\prime}=-y^{\prime 2}-z^{\prime 2}-a x^{\prime}+a(F+\sin (2 \pi \gamma t))$

$\dot{y}^{\prime}=x^{\prime} y^{\prime}-c y^{\prime}-b x^{\prime} z^{\prime}+G+\alpha p$

$\dot{z^{\prime}}=x^{\prime} z^{\prime}-c z^{\prime}+b x^{\prime} y^{\prime}+\alpha q$

$\dot{p}^{\prime}=-\omega q^{\prime}-\beta y^{\prime}$

$\dot{q}^{\prime}=\omega p^{\prime}-\beta z^{\prime}$

where the primed system $\boldsymbol{x}^{\prime}=\left(x^{\prime}, y^{\prime}, z^{\prime}, p^{\prime}, q^{\prime}\right)^{T}$ is identical to the original fully coupled system $\boldsymbol{x}=(x, y, z, p, q)^{T}$ except for slightly different initial conditions and the substituted variables $p$ and $q$ instead of $p^{\prime}$ and $q^{\prime}$, that emulate the forcing through prescribed data. In this system $p^{\prime}$ and $q^{\prime}$ have no influence on the dynamics of the other primed variables and are only introduced to allow for a closed math-

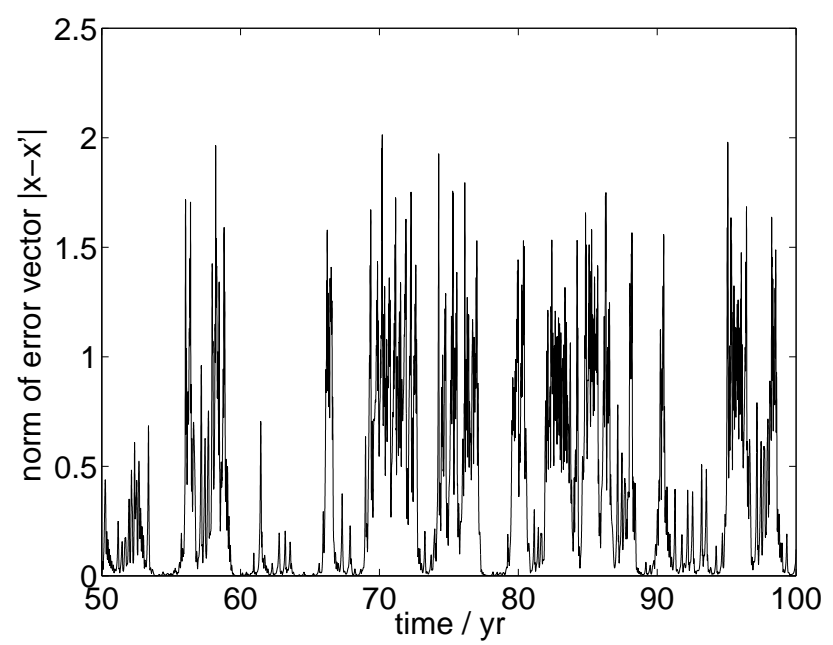

Fig. 2. Norm of the error vector $\delta \boldsymbol{x}=\left|\boldsymbol{x}-\boldsymbol{x}^{\prime}\right|$ between the fully coupled and the forced run; $(a=0.09)$.

ematical form. With this formalization "reality" - prescribed through data - is being represented through a perfect model scenario in the model-world.

In Fig. 2 the norm of the error vector $\delta \boldsymbol{x}=\left|\boldsymbol{x}-\boldsymbol{x}^{\prime}\right|$ is plotted. Sometimes the two systems synchronize but then suddenly the system shows long-lasting bursts, where the two trajectories seem to evolve independently.

Generally, this type of coupling between two identical systems can be written as

$\dot{\boldsymbol{x}}=\boldsymbol{F}(\boldsymbol{x}) \quad \dot{\boldsymbol{x}^{\prime}}=\boldsymbol{F}\left(\boldsymbol{x}^{\prime}\right)+\boldsymbol{K}\left(\boldsymbol{x}-\boldsymbol{x}^{\prime}\right)$,

where $\boldsymbol{K}$ is the coupling function.

By transforming Eq. (11) to the transversal coordinates $\boldsymbol{x}_{\perp}=\boldsymbol{x}-\boldsymbol{x}^{\prime}$ and considering only small perturbations, so that $\boldsymbol{x} \approx \boldsymbol{x}^{\prime}$ and $\boldsymbol{F}\left(\boldsymbol{x}^{\prime}\right) \approx \boldsymbol{F}(\boldsymbol{x})+\mathbf{J}(\boldsymbol{x})\left(\boldsymbol{x}^{\prime}-\boldsymbol{x}\right)$ the equation can be approximated by

$\dot{\boldsymbol{x}}_{\perp}=\boldsymbol{F}(\boldsymbol{x})-\boldsymbol{F}\left(x^{\prime}\right)-\boldsymbol{K}\left(x_{\perp}\right) \approx \mathbf{J}(x) x_{\perp}-\boldsymbol{K}\left(x_{\perp}\right)$,

where $\mathbf{J}(\boldsymbol{x})$ is the Jacobian matrix of $\boldsymbol{F}$ evaluated on the synchronization manifold. A linearisation of the function $\boldsymbol{K}(\boldsymbol{x})$ around zero, where we assume that $\boldsymbol{K}(\boldsymbol{0})=\boldsymbol{0}$ and neglect higher order terms of $\boldsymbol{x}_{\perp}$, leads to

$\dot{\boldsymbol{x}}_{\perp} \approx(\mathbf{J}(\boldsymbol{x})-\tilde{\mathbf{K}}) \boldsymbol{x}_{\perp}$,

with

$\tilde{\mathbf{K}}=\left.\frac{d \boldsymbol{K}}{d \boldsymbol{x}}\right|_{\boldsymbol{x}=0}$.

In our case, where we have linear coupling, the matrix $\tilde{\mathbf{K}}$ is

$\tilde{\mathbf{K}}=\left(\begin{array}{lllll}0 & 0 & 0 & 0 & 0 \\ 0 & 0 & 0 & \alpha & 0 \\ 0 & 0 & 0 & 0 & \alpha \\ 0 & 0 & 0 & 0 & 0 \\ 0 & 0 & 0 & 0 & 0\end{array}\right)$. 


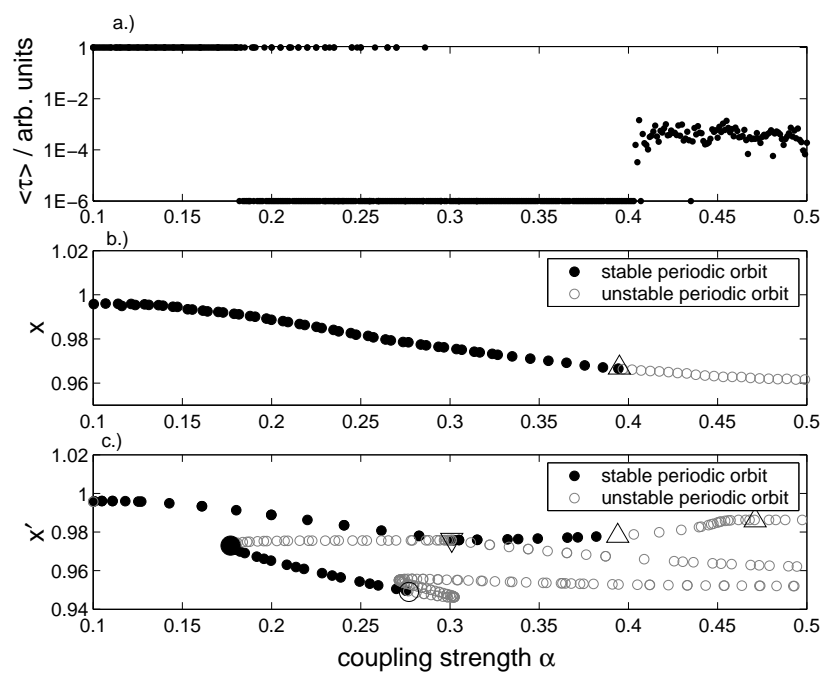

Fig. 3. Relative mean locking time in relation to the most dominant invariant sets (periodic orbits) for the system without seasonal cycle (with $a=0.12$ ). (a) Relative mean locking time $<\tau>$ (see Eq. 17) in dependence of the coupling strength $\alpha$. For every point in this figure the initial conditions for $\boldsymbol{x}$ were chosen randomly and the trajectories were integrated over 500 years, according to 730500 time steps, after they settled down on an attractor. The forced trajectory was started on the attractor with slightly perturbed initial conditions, chosen from a gaussian distribution with a standard deviation of 0.01 . For every value of the coupling strength $\alpha$ the integration was performed several times. As a mean length of zero cannot be depicted in a logarithmic plot, we added an offset of $10^{-6}$; (b) Bifurcation diagram for the variable $x$ of the five dimensional (5-D) driving system; for the periodic orbits, just one point referring to the maximum of the orbit is plotted; (c) Bifurcation diagram for the variable $x^{\prime}$ of the 8-D combined drive and response system. A filled circle symbol represents a saddle node bifurcation, an unfilled circle stands for a torus bifurcation, an upward-pointing triangle denotes a period doubling bifurcation and a downward-pointing triangle symbolizes a branch point.

To achieve complete synchronization, it is required that for $t \rightarrow \infty, \boldsymbol{x}_{\perp}$ goes to zero. From the linearised Eq. (13) one would expect that the two systems will synchronize if the transverse Lyapunov exponents, that are the Lyapunov exponents associated with Eq. (13), are all negative. This criterion was first proposed by Fujisaka and Yamada (1983), but in contrast to this, e.g. Gauthier and Bienfang (1996) observe only incomplete synchronization in their model instead of the proposed full synchronization, when the largest transverse Lyapunov exponent is smaller than zero. Several criteria for synchronization were developed (Blakely et al., 2000), but it was also shown there that for their model none of these criteria exactly predicts the range of the control parameter, where full synchronization can be observed.

In our case the largest transverse Lyapunov exponent is positive with $\lambda_{\perp} \approx 0.084$ but we also observe partial synchronization. The time $t^{*}$ after which all information is lost and the two trajectories are totally independent, reads

$t^{*} \approx \frac{1}{\lambda} \ln \frac{L}{\delta x(0)}$,

where $\lambda$ is the Lyapunov exponent, $L$ denotes the characteristic length of the attractor and $\delta x(0)$ the error that cannot be dissolved by a given accuracy (Argyris et al., 1995). Here $t^{*}$ is found to be about 1.3 years. Nevertheless locking can be observed over much longer timescales, as can be deduced from Fig. 2. This demonstrates that in the period of locking, a non-average, non-standard situation is present. Below we will link it to phase-space structures of low measure, yet of a noticeable domain of attraction.

\section{Comparison of forced and coupled system}

In this section we systematically compare the coupled to the forced system with respect to time-series properties as well as phase space topology. As a necessary condition for the forced system to emulate the coupled one in the time-domain we require that the forced system shows locking if and only if its coupled counterpart does.

\subsection{System without seasonal cycle}

An important structural difference between coupled and forced systems will be discussed in this section. In order to separate the two forcing effects in this model, namely the ocean forcing through the variables $p$ and $q$ and the seasonal forcing, the model is firstly investigated without the seasonal cycle. We analyse the dependence of the relative mean locking time $<\tau>$ on the coupling strength $\alpha$, see Fig. 3a, where

$\tau=\frac{t_{\text {locking }}}{T}$,

where $T$ is the length of the whole time series and $t_{\text {locking }}$ is the time, where locking can be observed. This is averaged over many locking periods. Locking is defined by the norm of the error vector $\delta \boldsymbol{x}=\left|\boldsymbol{x}-\boldsymbol{x}^{\prime}\right|$ of the two trajectories $\boldsymbol{x}$ and $\boldsymbol{x}^{\prime}$ being smaller than a critical threshold $\epsilon$. For a propper choice of $\epsilon$ see below, here we took $\epsilon=0.01$.

A significant difference in the relative mean locking time for a fully coupled run and a forced run can be observed. The fully coupled system consists of two totally independent systems $\boldsymbol{x}$ and $\boldsymbol{x}^{\prime}$, where the coupling matrix $\tilde{\mathbf{K}}$ of Eq. (15) is zero. The forced system is the 8-D combined drive and response system, consisting of Eqs. (1)-(5) and (6)-(8), the Eqs. (9) and (10) are neglected in this case as they have no influence on the system's dynamics. Whereas in the fully coupled system the relative mean locking time $\langle\tau\rangle$, as a function of $\alpha$, is always zero (not plotted in the diagram), in the forced system there are small parameter ranges, where the trajectories always show locking (for $\alpha \in[0.1,0.177]$ ), or where locking never appears, e.g. for $\alpha \in[0.277,0.4]$. Additionally, in the forced system there are also regions, where we observe intermittent synchronization as shown in Fig. 2, e.g. for $\alpha>0.4-$ like in the original system which includes a 


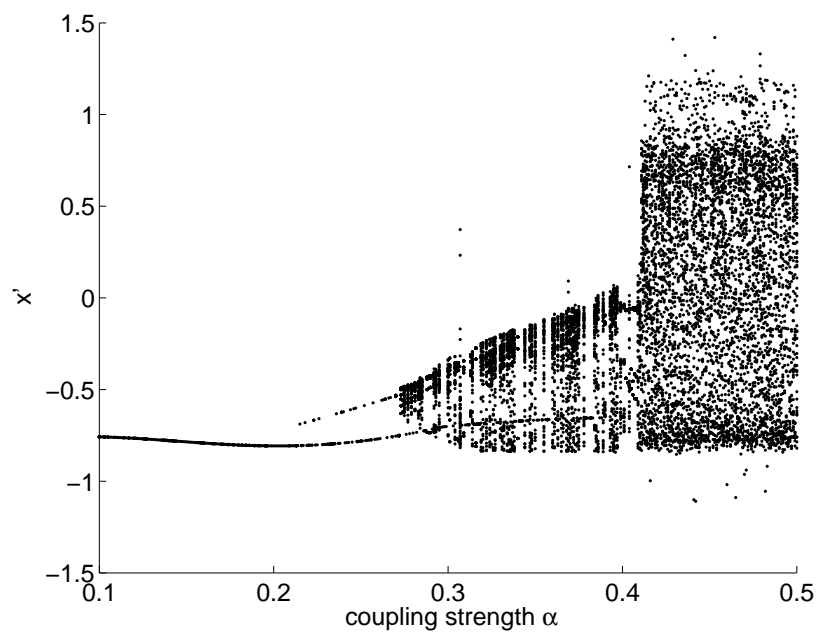

Fig. 4. Bifurcation diagram for $x^{\prime}$ in dependence of the coupling strength $\alpha$ in the system without seasonal cycle and $a=0.12$.

seasonal cycle. This suggests that the seasonal forcing is not the main cause of the observed intermittent behaviour.

The presented result does not depend on the choice of the threshold $\epsilon$, provided that $\epsilon$ is not too small. The relative mean locking time $\langle\tau\rangle$ grows only slowly with the $\epsilon$, so there would be only a slight shift for the value of $\langle\tau\rangle$ in Fig. 3a. On the other hand it is important to choose a threshold $\epsilon$ that is not too small (Lai, 1996), because it then takes a long time until the trajectory falls below the threshold and therefore one would need very long runs to calculate a reliable value for the mean locking time $<\tau>$.

In the remaining part of this section, we empirically correlate time-series properties and phase-space topology in order to explain the locking phenomenon of the forced system. To get an impression of the phase space topology of the system in dependence of the parameter $\alpha$, a bifurcation analysis is performed with the bifurcation analysis program AUTO (Doedel, 1981). In Fig. 3b the bifurcation diagram for the variable $x$ of the fully coupled system - which simultaneuosly plays the role of the forced system's master trajectory - is plotted, in Fig. 3c the same is done for the variable $x^{\prime}$ of the 8-D combined drive and response system. (Remember that for locking to occur, $x$ and $x^{\prime}$ must coincide in the time series.) For $\alpha<0.177$, both diagrams display the identical bifurcation diagram that simply consists of one stable periodic orbit. We propose that locking occurs in the forced system if both the master and the perturbed trajectory end up on the same periodic orbit.

For $\alpha>0.177$, the difference of the bifurcation diagrams is amazing: while for any $\alpha<0.4$ a stable periodic orbit exists in both the coupled (Fig. 3b) and the forced system (Fig. 3c), this orbit either lives on a different branch (for $\alpha \in[0.3,0.4]$ ) or another stable periodic coexists (for $\alpha \in[0.177,0.277]$ ), born in a saddle node bifurcation at $\alpha=0.177$. In the latter situation $(\alpha \in[0.177,0.277])$, full synchronization can be observed if the perturbed trajectory starts in the domain of
Table 1. Overview over the different regions in parameter space. SPO stands for stable periodic orbit, UPO for unstable periodic orbit.

\begin{tabular}{llll}
\hline $\begin{array}{l}\text { coupling } \\
\text { strength } \\
\alpha \in\end{array}$ & $x$ & $x^{\prime}$ & locking? \\
\hline$[0.1,0.177]$ & SPO & SPO & locking \\
\hline$[0.177$, & SPO & two SPOs & $\begin{array}{l}\text { locking, when } x \text { and } x^{\prime} \\
\text { are on the same PO; } \\
\text { else no locking }\end{array}$ \\
\hline$[0.277]$ & & & $\begin{array}{l}\text { locking, when } x^{\prime} \text { is } \\
\text { on the PO; no locking, } \\
\text { when } x^{\prime} \text { is on the torus }\end{array}$ \\
\hline$[0.3,0.4]$ & SPO & $\begin{array}{l}\text { (differing) } \\
\text { SPO and } \\
\text { torus }\end{array}$ & $\begin{array}{l}\text { no locking } \\
\text { and torus }\end{array}$ \\
\hline $0.4,0.5]$ & UPO & UPOs & $\begin{array}{l}\text { intermittent locking } \\
\text { (on-off synchroniza- } \\
\text { tion) }\end{array}$ \\
\hline
\end{tabular}

attraction of that periodic orbit which mimics the orbit of the master system (upper branch); otherwise the perturbed trajectory gets trapped by the concurring (lower branch) periodic orbit and locking never occurs. In the former case (for $\alpha \in[0.3,0.4])$, the attracting orbits differ, hence synchronization should be impossible. This is in fact consistent with the relative mean locking time observed in Fig. $3 \mathrm{a}$.

In Fig. 4 another bifurcation diagram for $x^{\prime}$ is obtained by numerical integration to capture the movement on a torus, that cannot be depicted in the other diagram. In fact, the torus bifurcation is found in the upper Fig. $3 \mathrm{c}$ for $\alpha=0.277$, but the torus cannot be followed with this method. For each parameter value $\alpha$ we let the system settle down to an attractor and then plotted the forced variable $x^{\prime}$, when the trajectory crosses the $y^{\prime}$ axis at 0.5 . From left to right we see again the stable periodic orbit and at $\alpha=0.177$ the generation of a second orbit. From these stable periodic orbits a quasiperiodic motion on a torus emerges at $\alpha=0.277$. The dynamics on the torus is sometimes adjourned by the stable periodic orbit that can be found in Fig. 3c for $\alpha<0.3$. The torus disappears at $\alpha=0.4$ through a period doubling bifurcation and passes into chaotic motion. For the driving system $\boldsymbol{x}$ no quasiperiodic dynamics are found, so that for $\alpha \in[0.277,0.3]$, before the branch point at $\alpha=0.3$ emerges, the system shows locking when $\boldsymbol{x}^{\prime}$ is on the stable limit cycle or shows no locking when $\boldsymbol{x}^{\prime}$ is on the torus. Which state will be adopted depends on the initial conditions. For $\alpha$ larger than the bifurcation value $\alpha=0.3$, there is scarcely any locking because the forced system is on the torus and the fully coupled system on the stable periodic orbit. An overview of the different classifications in dependence on the parameter space is given in Table 1. 


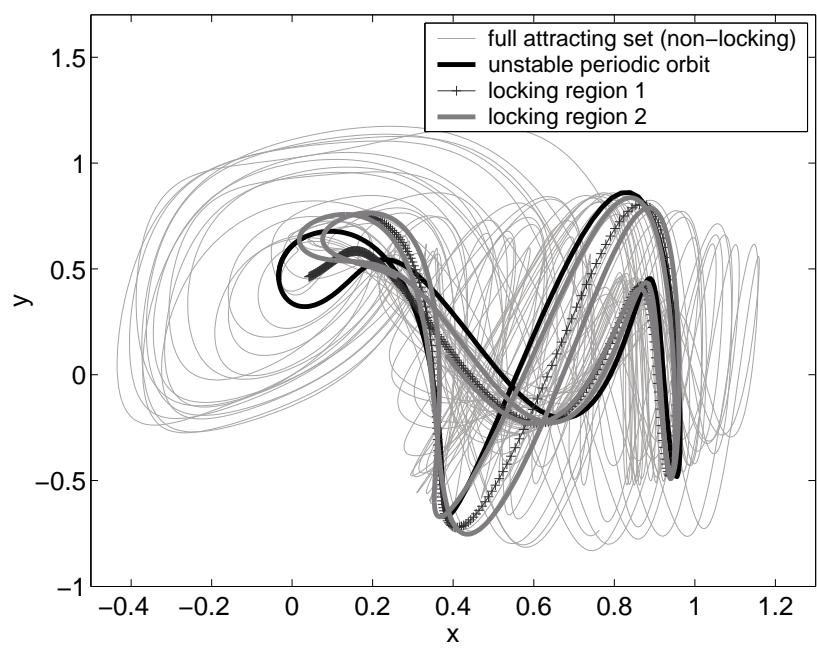

Fig. 5. Phase space of the full attracting set (non-locking region) and of two exemplary locking regions (1 and 2), whereas here region refers to a period in time. For comparison the unstable periodic orbit for this parameter constellation is plotted.

So far we can conclude that if the system is on the same stable periodic orbit for $\boldsymbol{x}$ and $\boldsymbol{x}^{\prime}$, we get full synchronization. On the other hand, when $x$ runs on a periodic orbit not concurring to the replicate of $\boldsymbol{x}$ 's periodic orbit, locking cannot be observed. That means there can be intrinsic obstacles that a forced system performs as the fully coupled system. For modelling issues this is a crucial outcome, as for more sophisticated models the calculation of the state space of the forced and the coupled model is very costly so there is hardly any way to decide if forcing is suitable, above all because normally the fully coupled model is not known. For users of forced models is therefore of central importance to note that synchronization effects might be artefacts from the forced set-up.

In order to obtain a full understanding of the fundamental discrepancies between the forced and fully coupled system, in the following we will focus on the phenomenon of intermittent synchronization that arises for $\alpha>0.4$, where no stable periodic orbit is detected.

\subsection{The role of (un)stable periodic orbits}

From the observations in the previous section we can conclude that if the system is in a region, where it is on a stable periodic orbit, the forced system shows locking all the time, provided that we are in a region in parameter space, where $\boldsymbol{x}$ and $\boldsymbol{x}^{\prime}$ show the same bifurcation diagram. The argumentation reads as follows: as the orbit of the 8-D system is stable, the trajectories of the driving system and the driven system end up on the same periodic orbit, but they could still have a phase shift. If there were a phase shift, then this shift would also be seen in the ocean coordinates. But this is excluded through the replica approach, where only the atmosphere coordinates are varied. Contrary to this, for the coupled system the ocean coordinates are also subject to the perturba- tion, hence no synchronizing drive is present and no locking will occur.

This emphasizes that full synchronization can in this case be explained by a stable periodic orbit (or a stable equilibrium point) that drives the forced system to the same dynamic behaviour.

After the stability has been lost in a bifurcation point, the unstable periodic orbit embedded into the attractor influences the system so that intermittently locking occurs even in a region, where the transversal Lyapunov exponent is positive and - naively, "on average" - no synchronization is expected. Therefore the concept of (unstable) periodic orbits seems to be crucial for the locking phenomenon and the loss of synchronization can be traced back to the transition from stable to unstable periodic orbits. Ott and Sommerer (1994) call this a "nonhysteretic" blowout bifurcation, where for $a<a_{c}$ the system is on an attractor and for $a>a_{c}$ on-off intermittency can be detected. The role of unstable periodic orbits (UPOs) for synchronization is also pointed out by Pazó et al. (2003) and by Pikovsky et al. (1997).

The interpretation with regard to UPOs can be stressed through Fig. 5, where we analysed the phase space of the locking regions in comparison to the full attracting set. It can clearly be seen that the locking region is in good coincidence with the unstable periodic orbit, whereas the non-locking region covers a much larger part of the whole phase space.

Just beyond a bifurcation point, where a periodic orbit has become unstable, the Monodromy matrix of the related mapping will display a long timescale on the unstable manifold, and generically shorter time-scales for the remaining stable manifold. Therefore, the unstable periodic orbit still has a fair chance to attract on the stable manifold and synchronize the trajectory. This will reveal itself as locking. After a while, the long timescale on the unstable manifold manifests itself, and the trajectory becomes repelled, reminiscent of intermittency. Hence, we suggest that the intermittent locking can be traced back to a co-existence of two identical unstable periodic orbits, one in the fully coupled, and one in the combined $8 \mathrm{D}$ fully coupled and replica system.

In summary, there are two major differences in the coupled and the forced system's dynamics: first, the forced system displays a richer bifurcation diagram including coexisting stable invariant manifolds, where the coupled system would not ("artificial bistability"), and even if both the master and the perturbed trajectory would end up on the same periodic orbit, the coupled system lacks a synchronizer, hence cannot display locking. At least the second effect is independent of the complexity of the model and should occur in GCMs as well.

One potential practical consequence could be within weather forecast: if locking occurred already at the beginning of the dynamics, in the "forecast period", the ensemble error along the trajectory could be vastly underestimated in forced ensembles. This could imply that e.g. a hurricane could affect a region on its way much earlier than anticipated.

Furthermore, artificial bistability could bring about that climate policy becomes overly conservative as society tries 

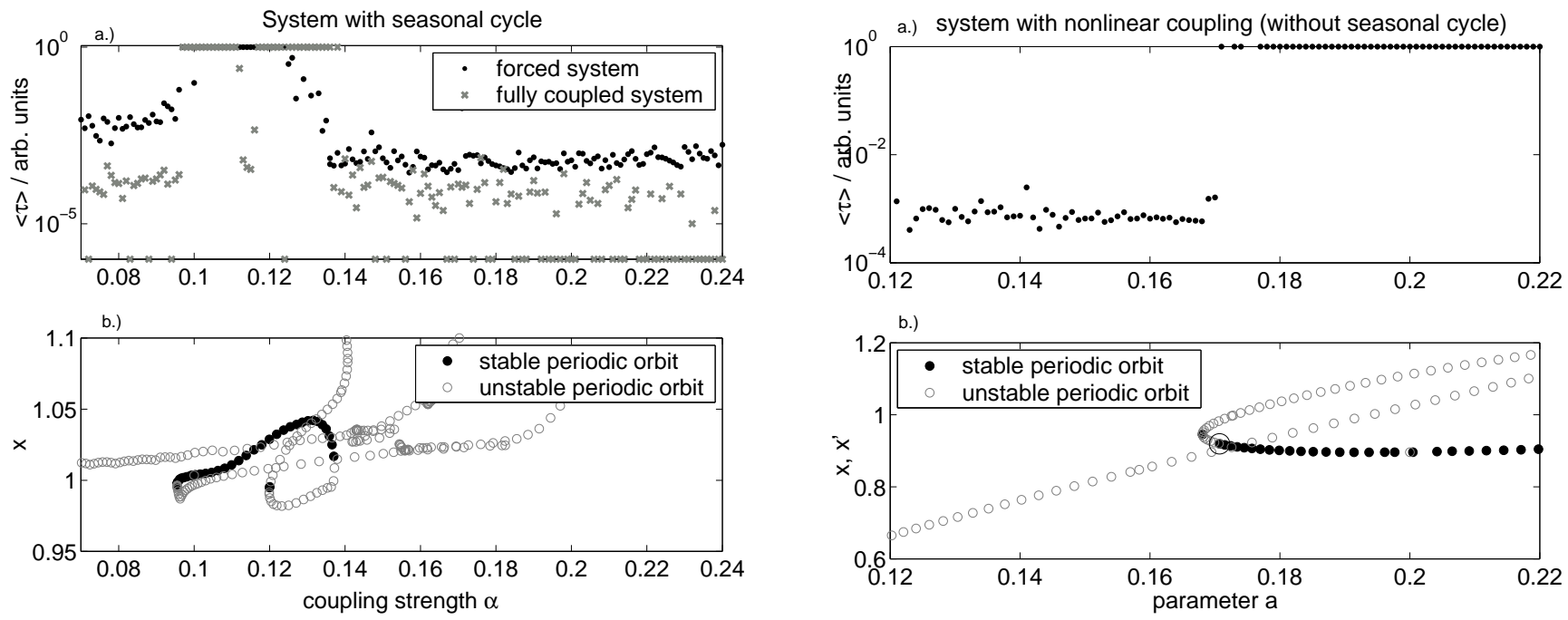

Fig. 6. Variation of the coupling strength $\alpha$ in the system with seasonal cycle with $a=0.27$. (a) Relative mean locking time $\langle\tau\rangle$ (see Eq. 17) in dependence on the coupling strength $\alpha$. Again we added an offset of $10^{-6}$ to depict a mean length of zero; (b) Bifurcation diagram of the system. As for this parameter constellation $x$ and $x^{\prime}$ show the same bifurcation diagram, just one bifurcation diagram is plotted.

to prevent crossing a threshold which is just an artefact from the forced model set-up.

\subsection{System with seasonal cycle}

The assertion of the role of stable and unstable periodic orbits can also be endorsed by Fig. 6, where the system with seasonal cycle in dependence on the coupling strength $\alpha$ is analysed. As before, the bifurcation diagram and the relative mean locking time are plotted. Again we can detect intermittent synchronization and it can be seen that there is a transition from locking to intermittent locking.

Figure 6 makes it clear that the presumption that with stronger coupling the two systems will synchronize, is not valid in this case. The system needs a stable manifold to become fully synchronized. For modelling issues, that means that it does not depend on the strength of coupling but on the state in the phase space if forcing can substitute coupling.

A significant difference to the situation without seasonal forcing is that here the fully coupled system shows locking when the system is on a stable limit cycle, see Fig. 6a, where the relative mean locking time is 1 in the locking regions, which means there is always locking. Intermittent synchronization can also sometimes be observed but less often than in the forced system (Fig. 6a). This is due to the seasonal forcing, that determines the frequency of the periodic orbit, so this locking bears on an external forcing and not on the intrinsic phenomenon of locking through prescribed forcing by variables. But as the seasonal cycle is also a kind of forcing, the chance of locking through an additional "synchronizer" increases.

Fig. 7. Influence of the parameter $a$ in the system without seasonal cycle and with nonlinear coupling as described through Eqs. (18) and (19). (a) Relative mean locking time $<\tau>$ (see Eq. 17) in dependence of the parameter $a$; (b) Bifurcation diagram of the 5-D and the 8-D system. Here $x$ and $x^{\prime}$ show the same bifurcation diagram. The unfilled cycle stands for a torus bifurcation.

\subsection{Influence of the type of coupling}

The system analysed so far is a system with linear coupling. As this is a very special case of coupling that is not often used in truly coupled models, we analyse a system without a seasonal cycle and with a nonlinear coupling to determine the influence of the type of coupling. The coupling has the following form:

$$
\begin{aligned}
& \dot{y}=x y-c y-b x z+G+\alpha p^{3} x \\
& \dot{z}=x z-c z+b x y+\alpha q^{3} x .
\end{aligned}
$$

instead of Eqs. (2) and (3). As $p$ and $q$ vary approximately between -1 and 1 , the introduced term bears strong nonlinearity.

Instead of analysing the influence of the coupling strength $\alpha$, here we focus on the effect of varying the parameter $a$. Again we have full locking when the system in on the same stable limit cycle for $x$ and $x^{\prime}$, and transitions to intermittent locking when an UPO is reached, see Fig. 7. By varying the coupling strength $\alpha$ in a range from 0.0 to 0.65 we discover a region of artificial bistability for $x^{\prime}$ (not shown here) as we have seen before in the system with linear coupling (Fig. 3).

So all features found in the linear coupled system can also be discovered in the system with nonlinear coupling. This demonstrates that the type of coupling (linear or nonlinear) has no decisive influence on the locking phenomenon. Quite the contrary, as periodic orbits appear frequently in nonlinear systems, locking may occur generically in forced systems and is much less likely in their fully coupled counterparts. This stresses that for the locking phenomenon a linear stability analysis does not hold. 


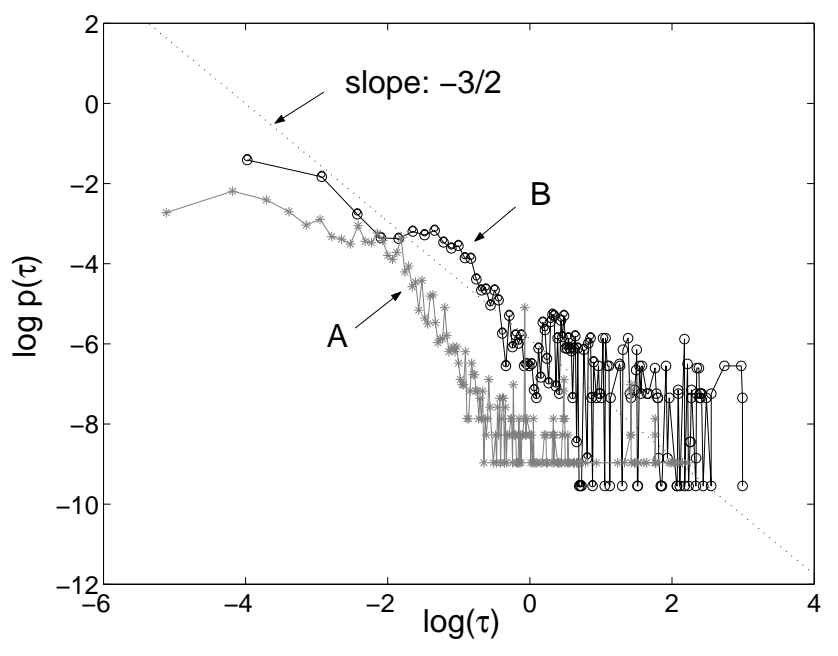

Fig. 8. Probability distribution of the length of the locking region for more than 14000 locking phases. Curve A is for a parameter setting as given following Eq. 5 with $a=0.125$, for curve B the parameter $a$ is changed to 0.075 , being closer to the bifurcation. The dotted curve indicates a $-3 / 2$ power-law for comparison.

\section{On-off synchronization}

The alternation between regions, where the error between the forced and coupled trajectory is nearly zero, and between regions with large bursts as shown in Fig. 2, resemble those of on-off intermittency. On-off intermittency, as introduced by Platt et al. (1993), refers to a situation, where the variables of a chaotic dynamical system exhibit two distinct states, where at the "off" state the system is nearly constant on an invariant manifold, and at the "on" state large bursts from these laminar phases occur. The frequency of bursts is controlled by a characteristic parameter $p$ of the system and approaches zero, when the so-called blowout bifurcation (Ott and Sommerer, 1994) is reached as $p$ attains a critical value $p_{c}$. To assign the phenomenon of on-off intermittency to our object of interest, intermittency is not seen as laminar phases interrupted by turbulent bursts but as locking of the fully coupled and the forced trajectories (off-state) interrupted by nonlocking (on-state), which we will call "on-off synchronization". The transfer from on-off intermittency to on-off synchronization becomes clearer when the difference between $\boldsymbol{x}$ and $\boldsymbol{x}^{\prime}$ is understood as a new variable.

Systems that generate on-off intermittency show characteristic scaling laws for the intermittent phases (Heagy et al., 1994; Lai, 1996), the distribution of the amplitudes of the bursts and for the power spectrum of the trajectories (see references cited by John et al., 2002). Heagy et al. (1994) investigate a certain class of driven systems, that consists of a discrete map and a random driving variable with a smooth density. They show that for the probability distribution of the length of the laminar phases $p(T)$ a power law holds with $p(T) \sim T^{-\gamma}$, where $T$ is the length of the laminar phases and $\gamma$ the scaling exponent that attains a universal value of $3 / 2$ in the vicinity of the threshold for on-off intermittency.
This scaling law with the same exponent can also be approved for our case of on-off synchronization. The probability distribution $p(\tau)$ for the length of the locking region $\tau$ for the parameter settings given in Fig. 1 (with $a=0.125$ ) is plotted as curve A in Fig. 8. As the $-3 / 2$ power-law distribution is deduced theoretically exactly only for the critical point $a_{c}$, we additionally plotted the probability distribution for $a=0.075$ in curve $\mathrm{B}$. This choice of the parameter is very close to the bifurcation point from a stable to an unstable periodic orbit at $a_{c}=0.073$, that was determined via bifurcation analysis.

As both curves indicate, when the parameter $a$ is close to the bifurctation point the probability distribution obey the predicted scaling law and differs from the exact relation when the parameter is further away from the critical value. For the very short locking phases both curves deviate from the predicted dependence, because one approaches the time scale of the simulation timestep. For the long locking phases the distribution for curve A falls off exponentially, whereas the two regions are connected by a shoulder, which is also seen in other systems displaying on-off intermittency (Platt et al., 1993). We assume that these imperfections from the exact scaling law is in our case due to the distance of the parameter $a$ from the bifurcation point.

In this section we have shown that the scaling law for the duration of the laminar phases in systems with on-off intermittency also holds for a system with on-off synchronization and could be extended to continuous systems with a driving system that is not random but chaotic. This means that the power law scaling is more universal than proposed when it was introduced. It can also be interpreted in this regard, that the underlying mechanisms of on-off intermittency and on-off synchronization are analogous. Intermittency is often traced back to the "almost existence" of a stable periodic orbit, and in a similar way we discovered stable and unstable periodic orbits as potential causes for locking.

The knowledge of the above power law does not only stress the underlying nature of locking but will also help in applications to estimate the relative importance of the locking phenomenon.

\section{Conclusions}

In this paper we consider the effect of module coupling on the overall dynamical uncertainty for a paradigmatic non-linear atmosphere-ocean system. We identify phase space as well as time-series features with respect to which a forced model set-up qualitatively differs from its fully coupled counterpart, for systematic reasons. On the one hand, in accordance with the general belief, the forced and the coupled model version coincide in various main features, in particular in terms of average predictive skill and the existence of the same dominant periodic orbit.

On the other hand, in fact we identify a considerable fraction in parameter space for which the phase spaces of the two model versions fundamentally differ: the phase space of the 
fully coupled model is dominated by a single stable periodic orbit, while the forced set-up allows for the existence of an additional stable periodic orbit. Since this kind of bistability is not found in the fully coupled model, which the forced set-up is supposed to emulate, we call it "artificial bistability". These finding seems to contradict conventional wisdom in the Earth System modelling community stating that a fully coupled model is more a complicated entity than a forced derivate, hence the coupled version is expected to display more complicated features. However, in terms of replica systems - a point of view we put forward in this paper - we argue that in fact the forced set-up is the more complex one: its dynamics are generated in an eight-dimensional (oceandimension plus two times the atmosphere-dimension) state space, while that of the coupled version resides in a fivedimensional space.

Furthermore, the systematic discrepancies of the two modelling versions extend into the time-domain. At least intermittently, the forced set-up displays artificial predictive skill. This is a direct consequence of the replica-nature of the forced set-up: we perturb the coordinates of the replica atmosphere in order to determine the predictive skill. As the perturbation cannot propagate to the five-dimensional subsystem driving the replica atmosphere, this five-dimensional sub-system potentially serves as a synchronizer. In case the replica atmosphere ("slave") and the synchronizer ("master") run in the vicinity of an identical periodic orbit which possesses a stable manifold, the ensemble will tend to collapse onto the master trajectory. Hence we identify the observed locking phenomenon as an almost-collapse to a periodic orbit. If the orbit is stable, locking will continue forever. If the orbit is unstable, the time-scale of locking is set by the competition of the stable and the unstable manifold of the periodic orbit, giving rise to intermittent locking. We observe a power law for the distribution of locking duration. Due to the phenomenological analogy to on-off intermittency, we call intermittent locking “on-off synchronization”. In any case, locking implies artificial predictive skill, which we explain by the existence of a partially attracting invariant set. For weather forecast this could imply that with a forced set-up, the ensemble error on the particular time, when a - potentially extreme - weather pattern will hit a certain region, can become extremely underestimated; hence an endangered region may prepare too lately.

All analysed features can be observed in the original system with seasonal forcing and linear coupling, in the system without seasonal forcing and finally in the system with nonlinear coupling. As we were able to explain these empirical findings with a universal theoretical pattern the ingredients of which just draw on the nonlinearity of the system, we suggest that on-off synchronization and artificial bistability are a general characteristic of forced systems rather than being restricted to this particular model set-up. While we expect that bifurcation analysis will be too demanding as a standard procedure for GCMs over the next years, nevertheless we advise that at least it is carefully checked with alternative model versions whether intermittent predictability and also bistability could not be the result of a forced - instead of the full-fledged coupled - set-up.

Acknowledgements. We would like to thank U. Feudel, J. Kurths, A. Pikovsky, and H. U. Voss for the fruitful discussions. This work was funded by the BMBF-projects 01LG0002 ("Model Validation and Ignorance Dynamics") and 07GCH02 ("Scientific Office for IGBP-GAIM"). B. Knopf acknowledges support from the Rosa Luxemburg Stiftung.

Edited by: G. Zöller

Reviewed by: two referees

\section{References}

Argyris, J. H., Faust, G., and Haase, M.: Die Erforschung des Chaos, Vieweg, 1995.

Barsugli, J. J. and Battisti, D. S.: The Basic Effects of AtmosphereOcean Thermal Coupling on Midlatitude Variability, Journal of Atmospheric Sciences, 55, 477-493, 1998.

Blakely, J. N., Gauthier, D., Johnson, G., Carroll, T., and Pecora, L.: Experimental investigation of high-quality synchronization of coupled oscillators, Chaos, 10, 738-744, 2000.

Boville, B. A. and Gent, P. R.: The NCAR Climate System Model, Version One, Journal of Climate, 11, 1115-1130, 1998.

Covey, C. K., AchutaRao, K. M., Cubasch, U., Jones, P., Lambert, S., Mann, M., Phillips, T., and Taylor, K.: An overview of results from the Coupled Model Intercomparison Project, Global and Planetary Change, 37, 103-133, 2003a.

Covey, C. K., AchutaRao, K. M., Gleckler, P., Phillips, T., Taylor, K., and Wehner, M.: Coupled ocean-atmosphere climate simulations compared with simulations using prescribed sea surface temperature: Effect of a "perfect ocean", Global and Planetary Change, 41, 1-14, 2003b.

Dijkstra, H. A.: Nonlinear Physical Oceanography, Kluwer Academic Publishers, Dordrecht, 2000.

Doedel, E. J.: AUTO: A program for the automatic bifurcation analysis of autonomous systems, in Proc. 10th Manitoba Conf. on Num. Math. and Comp., Univ. of Manitoba, Winnipeg, Canada, pp. 265-284, 1981.

Farrell, B. F. and Ioannou, P. J.: Generalized stability theory. Part I: autonomous operators, Journal of Atmospheric Sciences, 53, 2025-40, 1996.

Fujisaka, H. and Yamada, T.: Stability theory of synchronized motion in coupled-oscillator systems, Progress in Theoretical Physics, 69, 32-47, 1983.

Gates, W. L., Boyle, J., Covey, C., Dease, C., Doutriaux, C., Drach, R., Fiorino, M., Gleckler, P., Hnilo, J., Marlais, S., Phillips, T., Potter, G., Santer, B., Sperber, K., Taylor, K., and Williams, D.: An Overview of the Results of the Atmospheric Model Intercomparison Project (AMIP I), Bulletin of the American Meterological Society, 73, 1962-1970, 1992.

Gauthier, D. J. and Bienfang, J. C.: Intermittent loss of synchronization in coupled chaotic oscillators: toward a new criterion for high-quality synchronization, Physical Review Letters, 77, 1751-1754, 1996.

Heagy, J., Platt, N., and Hammel, S.: Characterization of on-off intermittency, Physical Review E, 49, 1140-1150, 1994.

Holton, J. R.: An introduction to dynamic Meteorology, Academic Press, 1992. 
John, T., Behn, U., and Stannarius, R.: Fundamental scaling laws of on-off intermittency in a stochastically driven dissipative patternforming system, Physical Review E, 65, 2002.

Lai, Y.-C.: Distinct small-distance scaling behavior of on-off intermittency in chaotic dynamical systems, Physical Review E, 54, 321-327, 1996.

Lorenz, E. N.: Irregularity: A fundamental property of the atmosphere, Tellus, 36A, 98-110, 1984.

Meehl, G., Boer, G., Covey, C., Latif, M., and Stouffer, R.: The Coupled Model Intercomparison Project (CMIP), Bulletin of the American Meterological Society, 81, 313-318, 2000.

Ott, E. and Sommerer, J.: Blowout bifurcations: the occurrence of riddled basins and on-off intermittency, Physical Letters A, 188, 39-47, 1994.

Pazó, D., Zaks, M. A., and Kurths, J.: Role of unstable periodic orbits in phase and lag synchronization between coupled chaotic oscillators, Chaos, 13, 309-318, 2003.

Pecora, L. M. and Carroll, T. L.: Synchronization in chaotic systems, Physical Review Letters, 64, 821-824, 1990.

Pecora, L. M., Carroll, T. L., Johnson, G. A., Mar, D., and Heagy, J. F.: Fundamentals of synchronization in chaotic systems, concepts, and applications, Chaos, 7, 520-543, 1997.
Pierce, D., Barnett., T., Schneider, N., Saravanan, R., Dommenget, D., and Latif, M.: The role of ocean dynamics in producing decadal climate variability in the North Pacific, Climate Dynamics, 18, 51-70, 2001.

Pikovsky, A., Zaks, M., Rosenblum, M., Osipov, G., and Kurths, J.: Phase Synchronization of Chaotic Oscillations in Terms of Periodic Orbits, Chaos, 7, 680-687, 1997.

Pikovsky, A., Rosenblum, M., and Kurths, J.: Synchronization: A universal concept in nonlinear sciences, Cambridge University Press, 2001.

Platt, N., Spiegel, E. A., and Tresser, C.: On-off intermittency: A mechanism for bursting, Physical Review Letters, 70, 279-282, 1993.

Smith, L. A., Ziehmann, C., and Fraedrich, K.: Uncertainty dynamics and predictability in chaotic systems, Quarterly Journal of the Royal Meteorological Society, 155, 2855-2886, 1999.

Wittenberg, A. T. and Anderson, J. L.: Dynamical implications of prescribing part of a coupled system: Results from a low order model, Nonlin. Proc. Geophys., 5, 167-179, 1998,

SRef-ID: 1607-7946/npg/1998-5-167.

Yanchuk, S., Maistrenko, Y., and Mosekilde, E.: Loss of synchronization in coupled Rössler systems, Physica D, 154, 26-42, 2001. 